\title{
Kernos
}

Revue internationale et pluridisciplinaire de religion grecque antique

6 | 1993

Varia

\section{Le mythe des races}

Une fiction aux sentiers qui bifurquent

\section{Marie-Christine Leclerc}

\section{(2) OpenEdition \\ 12 Journals}

\section{Édition électronique}

URL : http://journals.openedition.org/kernos/546

DOI : $10.4000 /$ kernos.546

ISSN : 2034-7871

Éditeur

Centre international d'étude de la religion grecque antique

\section{Édition imprimée}

Date de publication : 1 janvier 1993

Pagination : 207-224

ISSN : 0776-3824

\section{Référence électronique}

Marie-Christine Leclerc, "Le mythe des races », Kernos [En ligne], 6 | 1993, mis en ligne le 07 avril 2011 consulté le 02 mai 2019. URL : http://journals.openedition.org/kernos/546 ; DOI : 10.4000/kernos.546 
Kernos, 6 (1993), p. 207-224.

\section{LE MYTHE DES RACES}

\section{UNE FICTION AUX SENTIERS QUI BIFURQUENT}

Dans un article publié pour la première fois en 1910, E. Meyer invite à prendre au sérieux l'introduction d'Hésiode au mythe des races, et notamment le vers 108 des Travaux et les Jours, "dieux et hommes mortels ont même origine», dont il défend l'authenticité contre Lehrs et $\mathrm{Rzach}^{1}$. Selon lui, seul le poète lui-même pouvait s'exprimer ainsi, aucun interpolateur n'étant susceptible de lire, dans la succession des races humaines, une telle signification. Quoi qu'on pense de cet argument, certainement moins solide que l'absence de tout fondement paléographique à l'athétèse ${ }^{2}$, le souci de prendre en compte les indications d'Hésiode paraît raisonnable, et ce sera ici le seul : il s'agira de déterminer ce qu'apprennent sur la signification et le statut du mythe des races les trois vers qui l'introduisent :

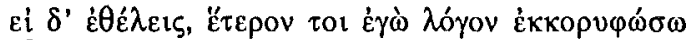

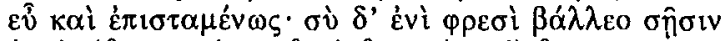

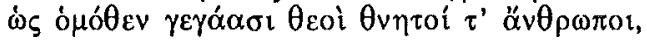

1 E. MEYER, Hesiods Erga und das Gedicht von den fünf Menschengeschlechtern, in Genethliakon. Festschrift der Graeca Halensis für Carl Robert zum 8. März 1910, Berlin, p. 157 sq., repris dans E. Heitsch (éd.), Hesiod, Darmstadt, 1966, p. 471-522 (ici p. 489-490); éditions K. LEHRs, Paris, 1840, A. RzACH, Leipzig, 1902; même athétèse dans l'éd. de P. Mazon, Paris, 1928, et déjà dans son commentaire aux Travaux, Paris, 1914, p. 60-61. Documentation complète sur le v. 108 dans J.-C. C ARRIÈre, Les mythes et les notions morales dans les Travaux et les Jours, thèse dactylographiée, Besançon, 1987, p. 859-865.

2 T.A. SinClaIr, Hesiod, Works and Days, éd. commentée, Londres, 1932 (rééd. 1979, p. 15); W. Nicolal, Hesiods Erga. Beobachtungen zum Aufbau, Heidelberg, 1964, p. 36 sq.; V. GolDSCHMIDT, Theologia, in REG, 63 (1950), p. 20 sq., repris dans Questions platoniciennes, 1970, p. 141-159, accompagné d'un «Addendum», p. 159-172 (ici p. 168); J.-P. VERNANT, Méthode structurale et mythe des races, in Histoire et structure. A la mémoire de V. Goldschmidt, Paris, 1985, p. 43-60 (ici p. 48). 
"si tu veux, je couronnerai mon récit par un autre, bien et savamment, et toi mets-toi bien en l'esprit que dieux et hommes mortels ont même origine» 3 .

\section{De quelques interprétations}

La critique s'est souvent écartée de la recommandation d'Hésiode pour expliquer le mythe par des données extérieures : recherche d'une origine orientale dont la succession des races semble tributaire ${ }^{4}$; recherche d'éléments de culte qui auraient pu inspirer Hésiode et justifier la présence, au sein d'un ordre fondé conjointement sur la succession des métaux et sur la décadence de l'humanité, de la race des héros qui en interrompt la logique. La double incongruité de cette race, qui n'est ni métallique ni pire que la précédente, mais au contraire "plus juste et meilleure» 5 avait autrefois suggéré à $\mathbf{E}$. Rohde l'idée que ce sont les héros et leur destinée posthume (ainsi que, secondairement, celle des autres races) qui se placent au centre de l'intérêt du poète ${ }^{6}$. En 1950 , V. Goldschmidt reprend "le principe» de cette interprétation : le mythe énumérerait, avec un effort marqué de systématisation, les puissances surnaturelles d'origine humaine auxquelles est rendu un culte : démons, héros, morts; il constituerait ainsi un complément à la Théogonie, qui ne traite que des dieux 7 .

Malgré sa cohérence et le crédit dont elle bénéficie, cette lecture se heurte à plusieurs objections.

3 Op., 106-108; le mythe occupe les vers 109-201.

4 Outre le dossier présenté par M.L. WEST, Hesiod. Works and Days. Edited with Prolegomena and Commentary, Oxford, 1978, p. 174-177, voir les études rassemblées par HEITSCH, op. cit., notamment celle de F. BRAMBERGER (1842), p. 439-449, R. Roth (1860), p. 450-470, R. REITZENSTEIN (1924), p. 523-544, A. HEUBECK (1955), p. 545-570, A. LESKY (1955), p. 571-601.

Op., 158.

6 E. RoHDe, Psyche. Seelencult und Unsterblichkeitsglaube der Griechen, Tübingen, 1907 (4e éd., p. 94-95). Selon G. NAGY, The Best of the Achaeans. Concepts of the Hero in Archaic Greek Poetry, Baltimore-Londres, 1979, p. 191, les races d'or et d'argent représenteraient les aspects des héros dans le culte, les deux races suivantes renvoyant aux aspects des héros dans l'épopée.

7 Goldschmidt, art. cit., p. 155. Voir également J. Defradas, Le mythe hésiodique des races. Essai de mise au point, in $I L$ (1965), 4, p. 152-156. 
1. Selon Goldschmidt, les hommes d'or et d'argent deviendraient après leur mort des "démons» ${ }^{8}$. Mais cette compréhension, si j'ose dire, technique du terme $\delta \alpha i$ inoves, qui nomme le statut posthume des hommes $d^{\prime}{ }^{9}{ }^{9}$, ne va pas de soi. Chantraine considère que ce sens n'apparaît qu'après Hésiode $^{10}$. Et il est de fait qu'aucun des deux autres emplois du terme par le poète ne confirme une telle interprétation. Au vers 314 des Travaux, $\delta \alpha i ́ \mu \omega v$ a sans ambiguïté le sens homérique de «divinité en général, destin». Le cas du demi-dieu Phaéton, promu par Aphrodite au

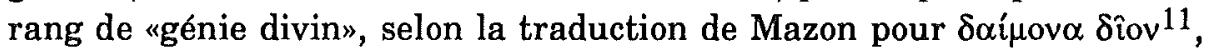
pourrait constituer un argument en faveur de Goldschmidt s'il n'entrait dans un catalogue où les destins des demi-dieux sont divers ${ }^{12}$ et où on ne trouve pas trace de la "systématisation" dont parle Goldschmidt. Celle-ci fait d'autant plus défaut qu'on voit un peu plus haut des demidieux promus au rang non de «démons" mais de $\theta$ coí13 et d'autres rendus «immortels» sans qu'un nom définisse leur statut ${ }^{14}$. On ne trouve, dans ce dossier, ni une taxinomie rigoureuse des puissances surnaturelles ni un usage rigoureusement fixe du mot $\delta \alpha i \mu \omega v$. C'est pourquoi je considère que Phaéton et les hommes d'or accèdent, par la grâce d'Aphrodite et par celle de Zeus, au statut de "divinités» en un sens indéterminé plutôt qu'au rang particulier de "génies» ou de «démons".

Ensuite, le poète n'appelle pas les hommes d'argent après leur mort

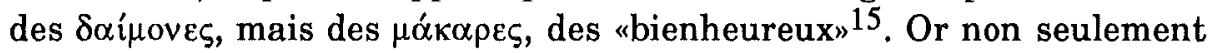
ce terme ne désigne pas un type de divinités déterminé, mais il s'applique aux dieux, au sens classique, dans le contexte immédiat ${ }^{16}$. On ne peut donc conclure purement et simplement à une catégorie

8 GoLDSCHMIDT, art. cit., p. 153-156.

9 Op., 122.

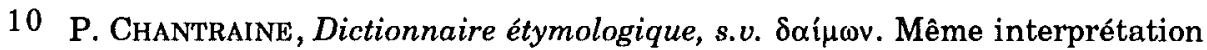
dans le commentaire de Mazon, 1914, p. 71.

$11 T h ., 991$.

12 Th., 965-1022.

$13 T h, 940-942$. In s'agit de Dionysos et de Sémélè.

14 Th., 949 (Ariane). GolDschmidT, art. cit., p. 155, n. 108, le remarque sans véritablement le prendre en compte.

15 Op., 141.

16 op., 136, 139. 
homogène de «démons», mais à des formes différentes de promotion des deux premières races au rang de «divinités».

2. L'adjectif vóvvuvor, appliqué aux hommes de bronze descendus dans l'Hadès, exclut que le poète songe à un culte funéraire ${ }^{17}$. Si, à l'inverse, il songe au culte en l'honneur des héros qui se développait, en effet, au VIIIe siècle avant notre ère ${ }^{18}$, il ne le dit pas et on ne peut raisonner comme si le texte apportait la preuve positive d'une telle préoccupation. D'autre part, l'adjectif $\theta \varepsilon \hat{\imath} o v$ appliqué à la race des héros ne signifie pas qu'ils ont une nature, un statut ou des honneurs divins, pas davantage que $\theta \varepsilon \hat{i} o \zeta$ n'a ce sens quand il s'applique à l'homme pudique au vers 731 des Travaux ${ }^{19}$. Rien de tout cela ne fait une hiérarchie systématique des puissances surnaturelles.

3. Si enfin, investi de la valeur que lui assigne V. Goldschmidt, le mythe des races était destiné à compléter la Théogonie, que viendrait-il faire entre les deux histoires qui l'encadrent, le mythe de Prométhée et la fable mettant en scène le rossignol et l'épervier? On ne voit ni comment une telle signification pourrait «couronner» l'épisode prométhéen, comme l'indique expressément la transition entre les deux mythes, ni plus généralement comment elle se rattacherait aux préoccupations des Travaux, où l'on ne trouve nulle trace de ces éventuels cultes. On ne voit même pas bien comment Goldschmidt concilie cette lecture avec la visée morale qu'il reconnaît lui-même au mythe $\mathrm{e}^{20}$.

L'autre grande interprétation moderne du mythe des races, non contradictoire avec la précédente aux dires des auteurs eux-mêmes, est celle de J.-P. Vernant. Selon l'«analyse structurale» qu'il propose dans les années 1960 , les races s'organisent par groupes de deux - la race de fer étant double - en un schéma trifonctionnel ${ }^{21}$. Les héros trouvent

17 Op., 154. La remarque est faite par VERNANT, art. cit., p. 55.

18 Voir notamment J.N. ColdSTREAM, Hero-Cults in the Age of Homer, in JHS, 96 (1976), p. 8-17; A.M. SNODGRASs, Archeology and the Rise of Greek State, Cambridge, 1977, p. 31.

19 VERNANT, art. cit., p. 49, qui formule la plupart des objections reprises ci-dessus sans pour autant rejeter la thèse de Goldschmidt.

20 GolDSCHMIDT, art. cit., p. 168-170.

21 J.-P. VERNANT, Le mythe hésiodique des races. Essai d'analyse structurale, in RHR (1960), p. 21 sq., repris dans Mythe et pensée chez les Grecs, Paris, 1965, 
ainsi tout naturellement leur place, en tant que bons guerriers opposés aux brutes de bronze, dans cette structure fortement charpentée. La valeur de cette interprétation est incontestable, mais partielle : on ne trouve pas plus trace de trifonctionnalité que de cultes dans les Travaux, et la race de fer, comme Vernant en convient maintenant, ne représente pas seulement la troisième fonction mais l'humanité actuelle dans son ensemble. Elle échappe donc à l'analyse structurale comme elle restait à l'écart de l'interprétation de Goldschmidt; Vernant suggère que le poète utilise cette structure comme «moyen» pour rendre clair le double diptyque moral qu'il entend mettre en place ${ }^{22}$ : l'opposition entre la "justice» et la "démesure» des quatre premières races, disposées en chiasme, se trouve ainsi déployée devant les yeux de la race actuelle ${ }^{23}$ comme un ensemble de modèles et contre-modèles, dont J.-C. Carrière a montré, dans des travaux récents, la fonction idéologique ${ }^{24}$ :

\begin{tabular}{|c|c|c|c|}
\hline or & argent & bronze & héros \\
\hline Síkn & üßpls & ïßpls & Síkn \\
\hline
\end{tabular}

Et un tel souci entre bien en effet dans l'intention du poète, qui reprend

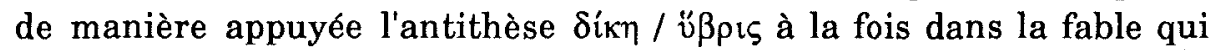
succède au mythe des races et dans les recommandations ultérieures ${ }^{25}$.

p. 19-47; Le mythe hésiodique des races. Sur un essai de mise au point, in $R P h$, 40 (1966), p. 247-276.

22 VERNANT, art. cit. (1985), p. 45-46.

23 Sur la disposition en chiasme, voir en particulier P. WALCOT, The Composition of the "Works and Days", in REG, 74 (1961), p. 4-7. Selon G. ARRIGHETTI (éd.), Esiodo. Le Opere e Giorni, Milan, 1985, p. 68, les quatre premières races, disposées sous les yeux des hommes actuels, ont valeur paradigmatique.

24 J.-C. CARRIÈre, Les démons, les héros et les rois dans la cité de fer. Les ambiguités de la justice dans le mythe hésiodique des races et la naissance de la cité, in Les grandes figures religieuses. Fonctionnement pratique et symbolique dans l'Antiquité, Paris, 1986 (Annales litt. de l'Univ. de Besançon, 329), p. 193 261, article comportant une excellente documentation bibliographique; Mystique ou politique dans Les Travaux et les Jours d'Hésiode. L'authenticité et les enjeux du vers 108, in Mélanges Etienne Bernand, Paris, 1991 (Annales litt. de l'Univ. de Besançon, 444), p.61-119. Dans son principe, la lecture "politique» de J.-C. Carrière n'est en rien incompatible avec celle que je propose ici et je partage ses analyses sur bien des points. Je ne crois toutefois ni qu'une partie des héros échappe à la mort (voir infra), ni que le vers 108 est une interpolation orphique.

Op. , 202-285. 
Ces interprétations retiennent également que le mythe des races, comme le mythe prométhéen qu'il "couronne», conte la séparation des hommes et des dieux à partir d'une origine commune ${ }^{26}$, mais paradoxalement elles relèguent au second plan ce niveau de signification, le seul que donne le poète, pourtant.

\section{Le processus de séparation}

Le mythe prométhéen des Travaux présente de manière ramassée ce que le mythe des races déploie dans la durée. "Avant» ( $\pi \rho i v$ ) que Pandora n'ouvre la jarre, les hommes vivaient «à l'écart et à l'abri des peines, de la dure fatigue, des maladies douloureuses qui apportent le trépas aux hommes",

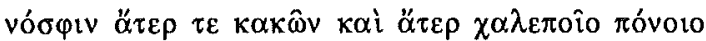

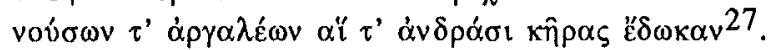

Après, les maux mortifères errent par le monde, agissant "d'eux-

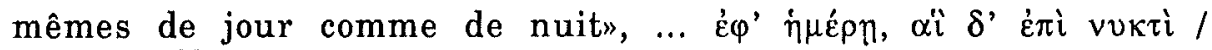

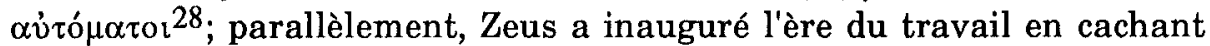
aux hommes leur "vie» 29 . A l'humanité pré-prométhéenne, correspond

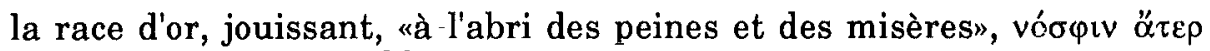

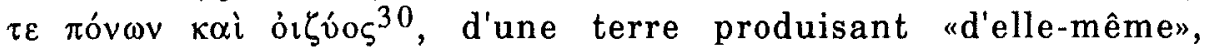
$\alpha$ $\tau_{\tau o \mu \alpha ́} \tau \eta^{31}$, ainsi que de félicités sans nombre. Les hommes de fer, aux prises «de jour» comme «de nuit" avec "fatigue et misères»,

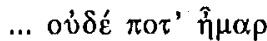

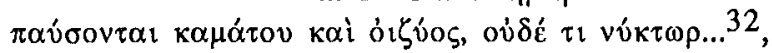

représentent l'état postérieur.

26 GoLDSCHMID', art. cit., p. 169; VERNANT, art. cit. (1985), p. 49-50. Les analyses de J. Fontenrose et de J. Rudhardt, sur lesquelles je reviens plus loin, prennent mieux en compte cette dimension de la signification.

27 Op. 90-92.

28 Op., 102-103.

29 Op. $42,47$.

30 Op., 113.

31 Op., 118.

32 op., 176-177. 
A quelques variantes près, le mythe prométhéen de la Théogonie conte la même histoire. Ce jour-là, "dieux et hommes se séparaient»,

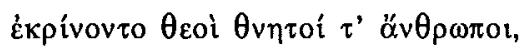

et «devant» eux Prométhée avait "placé», $\pi \rho \circ \theta \theta \eta \varepsilon \varepsilon$, le bœuf dépecé, avant que Zeus furieux n'envoie la femme «là où étaient dieux et hommes»,

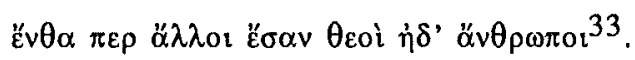

Jusqu'alors ils vivaient donc ensemble.

Le poète ne dévoile pas le contenu de cette coexistence, mais on peut en reconstituer quelques bribes. D'abord les hommes sont cités dès les premiers temps du monde, comme si leur existence, dont aucun mythe ne vient relater l'origine, allait de $\mathrm{soi}^{34}$. Ensuite les appellations d'«homme» et «mortel», qui ne sont pas exactement synonymes ${ }^{35}$, ne convergent que tardivement dans un passage où il arrive aux dieux de mourir. Certains descendants de Phorkys et Kèto, fils et frères de dieux immortels et dieux eux-mêmes, sont en effet tués ${ }^{36}$. La première d'entre eux est Méduse, «mortelle» soeur de «deux immortelles»,

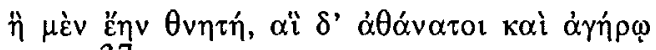
$\alpha \mathrm{i} \delta \mathrm{vó}^{37}$.

Un tel sort est inexplicable si on ne suppose pas que les puissances létales issues de Nuit, nées quelques dizaines de vers auparavant sans qu'on les ait encore vues à l'œuvre, s'abattent à l'étourdie faute d'une délimitation claire de la catégorie de "mortels». Celle-ci se fixe sur l'espèce humaine quelques vers après le meurtre de Méduse, dans une expression qui associe pour la première fois l'adjectif «mortel» au nom «homme»,

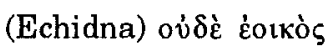

$33 T h, 535,586$.

34 Les hommes sont cités dans l'énumération des puissances primordiales (Chaos, Gaia, Tartare, Éros), cinq vers après le début de la théogonie proprement dite (Th., 121).

35 MAZON, 1914, p. 72, attire l'attention sur ce point. Voir également les remarques d'I. SELLSCHOPP, Stilistische Untersuchungen zu Hesiod, Hambourg, 1934, p. 24-25.

36 Th., 270-336.

37 Th., 277-278. 


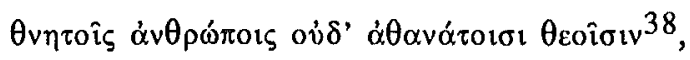

alors que l'immortalité des dieux a été indiquée depuis longtemps. Toutes ces bizarreries suggèrent que, si l'immortalité est originelle, la mortalité est en revanche un caractère acquis dont cette descendance conte la mise en place.

Avec le mythe de Prométhée, qui totalise à lui seul la moitié des emplois de l'expression «hommes mortels» figurant dans la Théogonie ${ }^{39}$, le processus s'achève par un récit dramatisé qui le rend explicite. Certes, le mythe conte l'émergence du mode de vie civilisé, comme Vernant l'a montré 40 . Mais ce mode de vie ne se conçoit que si les hommes sont séparés des dieux, soumis aux contraintes de l'alimentation et de la reproduction, c'est-à-dire s'ils sont mortels. Le responsable de leur sort est Zeus, par qui tout arrive. Quand Prométhée l'invita à choisir entre les deux tas du bœuf dépecé, Zeus "comprit la ruse et ne l'ignora pas»; deux vers plus loin, il choisit le tas d'os, délibérément donc. Entre ces deux passages à l'aoriste, Hésiode insère une remarque à l'imparfait :

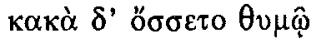

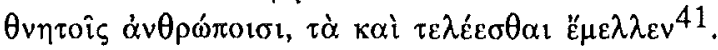

Mazon et Hofinger comprennent que Zeus "méditait la ruine des mortels tout comme en fait il devait l'achever» 42 . Je ne crois pas que le

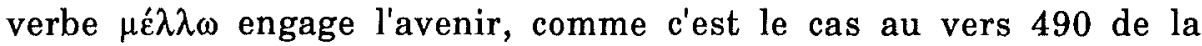
Théogonie, où il est employé avec $\tau \dot{\alpha} \chi \alpha$. Ici, l'adverbe fait défaut, et rien n'interdit de comprendre le verbe dans son sens le plus courant 43 : Zeus ruminait des maux «qu'il était sur le point d'achever». Le partage du bœuf lui en fournit l'occasion sans en être la cause efficiente. Le processus ne trouve à Mékonè que son achèvement, sinon les imparfaits ne se justifieraient pas.

$38 T h ., 295-296$.

39 Th., 535, 552, 564, 588, 592, 600.

40 J.-P. VERNANT, "Le mythe prométhéen chez Hésiode», in Mythe et société en Grèce ancienne, Paris, 1974, p. 177-194.

41 Th., 551-552.

42 MAzon, 1928, p. 52; HofINGER, Lexicon hesiodeum, Leyde, 1975-1978, s.v. $\mu \varepsilon ́ \lambda \lambda \omega$.

$43 T h_{n}, 468,478,888,898$. 
À cette trace presque effacée de l'histoire des hommes, le mythe des races substitue un récit détaillé dont plus d'un élément concorde avec la reconstitution ci-dessus proposée. Les hommes d'or représentent l'époque antérieure au processus de séparation. Ils «étaient du temps de Cronos", ce qui signifie de temps très anciens; «ils vivaient comme des dieux", et participaient à des "fêtes» ou "festins" qui figurent plus d'une fois la félicité divine ${ }^{44}$. Ils "mouraient" cependant, mais sans avoir vieilli et comme s'ils s'endormaient, emportés par un trépas si différent

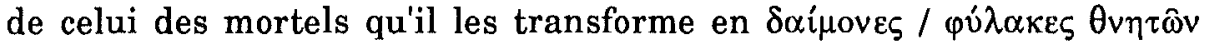
$\dot{\alpha} v \theta \rho \dot{\omega} \pi \omega v$, «divinités gardiennes des hommes mortels", que les Travaux, plus loin, diront $\dot{\alpha} \theta \alpha \dot{\alpha} v \alpha \mathrm{o}^{45}$.

Les hommes d'argent se sont éloignés des dieux, dont ils constituent sans doute un groupe distinct puisqu'ils devraient leur rendre un culte $^{46}$. Le cortège de Nuit les a sans doute précédés : ils connaissent le mal à tel point que c'est la seule race dont il est dit qu'elle provoque la "colère» de Zeus ${ }^{47}$. Aussi est-il paradoxal qu'une promotion au rang de

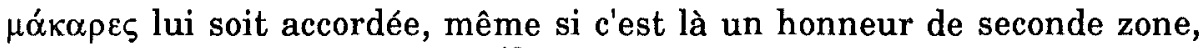

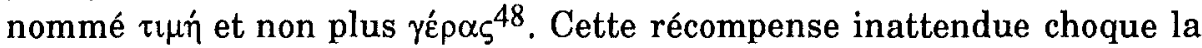
morale, mais l'étrange groupe $\mu \alpha \alpha \kappa \alpha \rho \varsigma \varsigma$ $\theta v \eta \eta$, souvent commenté ${ }^{49}$, permet probablement de lui donner un sens. Même si le premier terme s'applique aux hommes dans un autre contexte ${ }^{50}$, il peut difficilement, après avoir désigné deux fois les dieux dans les cinq vers précédents, admettre une acception différente dans cette dernière expression. En réalité, les hommes d'argent, «bienheureux» mais "mortels», semblent caractérisés par le même type d'ambiguité que Méduse, inexplicablement déesse et «mortelle» à la fois. Ils sont de cette époque où la mort

$44 O p ., 112,115$; également fragment 1 (Merkelbach-West). Sur le sens de $\theta \alpha \lambda i$ in comme «fête", J. Duchemin, Pindare, poète et prophète, Paris, 1955, p. 55. Sur le festin infini comme figure de la félicité divine, G. Sissa, in G. SIsSA, M. Detienne, La vie quotidienne des dieux grecs, Paris, 1989, p. 98.

Op., 250, 253.

Op., 135-136.

48 Comparer $O p ., 126$ et 142.

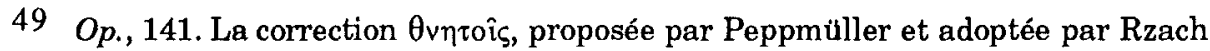
et Mazon, ne se justifie pas. Voir les arguments de SiNCLAIR, op. cit., p. 19, et de WEST, op. cit., p. 186-187, ainsi que l'analyse de VERNANT, art. cit. (1985), p. 5455. 
rôde sans que les modalités de la mortalité soient encore définies. Ces coups d'essai des puissances létales ont sans doute quelque rapport avec les fantaisies du temps, qui, laissant les hommes d'argent dans l'enfance jusqu'à leur mort, ne parvient guère à s'écouler 51 .

La mort véritable arrive avec les hommes de bronze. Ils sont les premiers à descendre dans l'Hadès. Celui-ci, ainsi que leur trépas exprimé trois fois, sont décrits dans les termes qu'Homère emploie pour dire la mort,

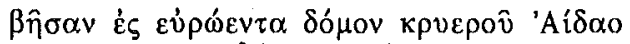

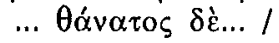

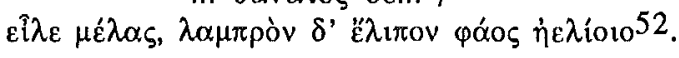

Comme dans l'épopée également, ils sont les «sans nom», les oubliés qui ne sont plus personne ${ }^{53}$. Par l'insistance qu'il y met et par le choix de tournures connues, claires pour l'auditoire, Hésiode fait de la race de bronze celle des mortels par excellence. S'ils «succombent sous leurs propres bras", c'est évidemment parce que ce sont des brutes guerrières ${ }^{54}$, mais c'est peut-être aussi une manière de dire qu'ils portent en eux-mêmes, sans qu'il soit besoin de les anéantir de l'extérieur, leur destin périssable. Sans doute est-ce pourquoi ils ont parfois été considérés comme les premiers hommes connus dans la tradition grecque ${ }^{55}$. Il faudrait ajouter : les premiers hommes mortels au sens plein du terme.

Les héros, bien qu'ils se distinguent des hommes de bronze par leur caractère meilleur et plus juste, ont avec eux deux points communs : ce sont des guerriers et ils meurent. L'exposé des circonstances de leur trépas et la description de leurs différentes situations posthumes constituent même le principal sujet du passage, où ils occupent treize vers sur dix-huit ${ }^{56}$. Les uns subissent le même sort que leurs

51 Op., 130-133.

52 Op., 153-155.

53 Op., 154. Yoir J.-P. VERNANT, «Mort grecque, mort à deux faces», in L'individu, la mort, l'amour, Paris, 1989, p. 81.-89.

54 Op., 152.

55 W EST, op. cit., p. 187.

56 Op., 161-173 sur l'ensemble 156-173. Je ne tiens évidemment pas compte de l'addition du papyrus de Genève, insérant dans le passage une tradition postérieure à Hésiode concernant les Titans. Voir à ce sujet MAzon, 1928, p. 92 et la note, et surtout WEST, op. cit., p. 194-195. 
prédécesseurs, victimes d'un trépas énoncé, lui aussi, dans des termes connus d'Homère ${ }^{57}$. Les autres, pour être accueillis aux Îles des Bienheureux et y trouver une abondance et une félicité qui rappellent les conditions de vie des hommes de la race d'or, n'en ont pas moins péri. Les Îles des Bienheureux, situées «aux confins de la terre... près des tourbillons profonds de l'Océan" 58 , ne sont pas fort éloignées des Enfers : ce lieu écarté, où Styx se sépare de son père Océan ${ }^{59}$, est «à la frontière de Nuit, ${ }^{60}$, qui croise Jour sur le seuil de sa maison ${ }^{61}$, voisine des «demeures d'Hadès» 62 . Le séjour des héros ne se distingue probablement pas de ce que l'Odyssée appelle "plaine Élyséenne» ou "prairie d'asphodèles", dont la situation est la même ${ }^{63}$ et que Platon utilise pour décrire les Îles des Bienheureux ${ }^{64}$. Ces zones de félicité n'abritent que des morts; elles sont comme un paradis qui récompense des mérites, non le séjour d'une catégorie d'immortels, et le texte d'Hésiode ne dit rien de tel. La séparation des hommes et des dieux, à l'époque des héros, est en outre confirmée par l'emploi du terme $\grave{\eta} \mu i^{\theta} \varepsilon_{0}{ }^{65}$, car si les hommes et les dieux ne constituaient pas deux groupes distincts, on ne pourrait parler entre eux de métissage. Au moment où va commencer la dernière partie du mythe, le processus de séparation des dieux et des hommes, au cours duquel ces derniers acquièrent le statut de mortels, est enraciné à la fois dans l'évolution du monde telle que l'a contée la Théogonie et, si j'ose dire, dans l'«histoire» des hommes telle que les héros de l'épopée la représentent.

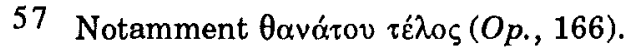

58 Op., 168-170.

59 Th., 787-789.

60 Th., 274-275.

$61 T h ., 748-754$.

62 Th., 766-767.

63 Od., IV, 561-569; XXIV, 13-14. Au chant XI, Ulysse évoque les morts en sacrifiant au bord de l'Océan (v. 13,539). Je ne crois pas, contrairement à CARRIÈre, art. cit., 1986 et 1991, que le cas de Ménélas (Od., IV) constitue une preuve suffisante pour affirmer qu'une partie des héros d'Hésiode échappe à la mort. Les données des chants XI (Nekuia) et XXIV de l'Odyssée vont en sens inverse.

64 Platon, Gorgias, 523a-524a.

65 Op., 160. 
Hésiode ne dit presque rien des hommes actuels ${ }^{66}$. Il peut y avoir à ce laconisme deux raisons qui ne sont pas contradictoires. D'une part, il a longuement évoqué la situation présente dans le mythe de Prométhée. D'autre part, si l'on en juge par la place qu'il lui accorde, c'est l'avenir de la race qui l'intéresse. La perversion morale qui régnera alors, et sur laquelle je ne reviens pas, est enveloppée par deux éléments convergents. $\AA$ la fin du mythe, Aidôs et Némésis quitteront la terre pour rejoindre les dieux ${ }^{67}$. C'est à la fois le résultat de l'immoralité des hommes et l'aboutissement logique du processus de séparation : rien ne reliera plus les deux groupes autrefois unis. D'autre part au début de l'évocation des futurs hommes de fer, on lit que les hommes «naîtront avec des tempes blanches» ${ }^{68}$. Le mythe a été jalonné d'indications physiques: les hommes d'or avaient la vigueur de la jeunesse; les hommes d'argent étaient de taille moins avantageuse; les guerriers de bronze terrifiaient par la force de leurs bras ${ }^{69}$. Dans tous ces cas, les traits corporels avaient un rapport direct avec le statut de la race en cause. L'état de ces nouveau-nés portant les traits de la vieillesse figure évidemment l'inversion des valeurs développée ensuite, mais il évoque aussi un renversement sur le plan physique. La mort n'est pas pour eux l'«achèvement» ( $\tau$ é $\lambda \circ \zeta)$ d'une durée de vie, comme il était dit pour les héros ${ }^{70}$, mais la matrice qui les marque «dès leur naissance», $\varepsilon \hat{\jmath} \tau$ ' ${ }^{\circ} v$

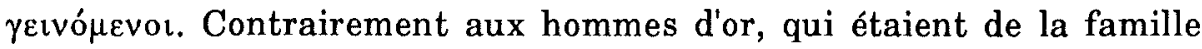
des dieux, ceux-ci vivront d'emblée dans la familiarité de la mort.

Hésiode prend donc appui sur le processus de séparation dont la Théogonie porte la trace pour en pousser jusqu'au bout la logique interne, et ceci de deux manières. Il y a d'abord une logique linéaire : à une humanité proche des dieux et tendant asymptotiquement vers l'immortalité, succèdent des espèces, si j'ose dire, de plus en plus mortelles, la dernière d'entre elles, abandonnée des dieux, tendant asymptotiquement vers la mort. Si c'est bien la logique interne du récit qui motive le tableau final, on ne doit pas le prendre tout bonnement pour une prophétie, et il est vrai que tout le poème s'efforce à convaincre qu'on

$\begin{array}{ll}66 & \text { Op., 176-179. } \\ 67 & O p ., 197-201 . \\ 68 & O p ., 181 . \\ 69 & O p ., 113-114,129,145,148-149 . \\ 70 & O p ., 166 .\end{array}$


peut éviter d'en arriver là. Il ne s'agit que d'un des avenirs logiquement possibles pour les mortels.

Dans un second cas de figure, logiquement possible aussi, leur sort est différent. Après l'ensemble formé par les trois premières races et le début de la quatrième (les héros simplement morts), qui suffit à constituer un mythe d'éloignement de l'immortalité, le récit suit une logique circulaire en revenant sur lui-même : la félicité aux Îles des Bienheureux ressemble à celle des hommes d'or. Mais il faut souligner qu'il s'agit dans ce dernier cas de récompenses posthumes décernées à une race juste. En effet la composition annulaire, que Walcot a bien vue $^{71}$, ne va pas jusqu'à faire coïncider totalement les héros avec les hommes d'or, car les héros ne connaissent pas cette félicité de leur vivant. Ils ne deviennent pas non plus des divinités après leur mort: le terme «bienheureux», repris à la race d'argent, ne renvoie pas, dans le contexte des héros, à l'octroi d'une $\tau \imath \mu \eta$, mais à la qualité d'un séjour qui rend la mort plus douce sans pour autant en dispenser. Je suis tentée de dire que la rigueur formelle de la "ring composition" donne autant de relief aux différences qu'aux ressemblances. L'évolution de la mortalité empêche l'anneau narratif de se refermer tout à fait, et le modèle ne peut donner lieu qu'à une reproduction décalée.

La visée morale du poète, soucieux de convaincre qu'il faut être juste, et sa description du processus de séparation s'articulent dans cette coexistence de deux schémas logiques apparemment inconciliables. Les hommes actuels qui, contrairement aux autres races, ne se caractérisent ni par la justice ni par le malheur absolu, mais le mélange de biens et de maux, se trouvent placés là où les chemins logiques bifurquent: immédiatement après la félicité aux Îles, immédiatement avant les horreurs à venir. J. Fontenrose et $J$. Rudhardt ont sans doute raison de penser qu'il y entre les deux dernières races une continuité plus étroite qu'entre les autres ${ }^{72}$. Car le poète ne dit ni que la "terre a recouvert» la race des héros, comme il le fait pour les trois premières races, ni que les hommes de fer furent

71 W ALCOT, art. cit.

72 J. Fontenrose, Work, Justice and Hesiod's five ages, in CPh, 69 (1974), p. 116; J. RudHARDT, "Le mythe hésiodique des races et celui de Prométhée. Recherche des structures et des significations", in Du mythe, de la religion grecque et de la compréhension d'autrui, Revue européenne des sciences sociales, XIX, 58, Genève, 1981, p. 255-257; VERNANT, art. cit. (1985), p. 60, n. 12 . 
"fabriqués" comme le sont les autres ${ }^{73}$. Suspendus entre l'âge antérieur, qui offre un modèle de justice et une promesse de récompense, et l'âge postérieur, qui en est l'inverse exact, les auditeurs d'Hésiode doivent choisir. La curieuse expression "plût au ciel que je fusse ou mort plus tôt ou né plus tard» ${ }^{74}$ pourrait témoigner, plutôt que d'une conception cyclique du temps ${ }^{75}$, de la confiance d'Hésiode dans un avenir qui, "hommes et dieux ayant même origine", reste ouvert à des évolutions positives ${ }^{76}$.

Il y a ce qui nous échappe : l'état dans lequel nous a mis l'évolution du monde voulue par les dieux; il y a ce qui nous revient: la manière dont nous disposons de cet état est de notre responsabilité.

\section{Les éléments de fiction}

Hésiode veut convaincre et, pour y parvenir, fait feu de tout bois. L'agencement de l'ordre chronologique explicite avec une visée morale, des architectures logiques, une matrice trifonctionnelle, relève d'une virtuosité dont le poète est conscient, lui qui va conter "savamment",

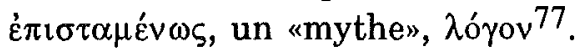

C'est la première fois qu'on trouve ce terme au singulier. Chez Homère et dans les autres emplois hésiodiques, il est question de $\lambda o ́$ or, dont l'acception est celle de la tromperie, soulignée par les déterminants, le contexte et le voisinage fréquent des "mensonges» 78 .

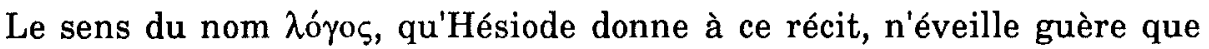
la curiosité de West, qui ne tient d'ailleurs plus compte de ses

73 Comparer $O p ., 110,127,144,158$ et 176.

74 Op., 174-175.

75 Cette interprétation a été reprise en dernier lieu par B. MEzzARDI, Structure du mythe et races d'Hésiode, in L'Homme, 106-107 (1988), p. 51-57, qui propose, pour rendre compte de l'expression, l'image du ruban de Möbius. L'idée est ingénieuse, mais l'argumentation n'est pas étayée sur une documentation assez solide.

76 Suggestion de T.G. RosEnMEYer, Hesiod und die Geschichtsschreibung, in Hermes, 85 (1957), p. 257 sq., repris dans HeITsch, op. cit., p. $602-648$ (ici p. 631-633, où l'on trouve une argumentation assez convaincante contre une conception cyclique du temps chez Hésiode).

77 Op., 106-107.

$78 T h ., 229,890 ; O p ., 78,789$, pour s'en tenir aux emplois hésiodiques. 
intéressantes remarques dans la suite de son commentaire ${ }^{79}$. Dans une étude récente, C. Calame a montré que l'opposition muthos-logos, devenue classique en philosophie, est moderne et non grecque : Platon et Aristote utilisent encore largement $\lambda$ ó ${ }_{0} \varsigma$ au sens de "fable» 80 . Le dictionnaire étymologique de Chantraine reconnaît au terme, entre autres acceptions, le sens de "fiction» 81 . Certains éléments du mythe des races pourraient confirmer son statut de récit fictif assumé comme tel par le poète.

D'abord la notion d'une fabrication des hommes par les dieux n'est ni grecque en général ${ }^{82}$ ni, en particulier, hésiodique. La Théogonie n'en souffle mot, cite les hommes dès les premiers temps du monde comme si de rien n'était et ne mentionne que la "séparation" des hommes et des dieux. Tout porte à croire que la vérité à "se mettre en l'esprit» est bien, en effet, qu' «hommes et dieux ont même origine»; les péripéties narratives en constitueraient une illustration figurée.

D'autre part, Hésiode connaît parfaitement le caractère symbolique des métaux dont ses hommes sont faits, comme Socrate le notait déjà : "mon opinion est que par "race d'or" il entend non pas "née de l'or", mais "bonne et belle". Et la preuve pour moi, c'est qu'il nous appelle

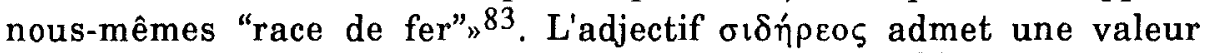
métaphorique lorsqu'il désigne la dureté d'un cœur ${ }^{84}$. C'est dans ce sens qu'il faut le comprendre ici, et il en va de même pour le bronze. Métal guerrier et funèbre, il passe pour clore les Enfers, dotés d'un seuil, de portes et d'un mur "de bronze», gardés par Cerbère «à la voix de bronze» ${ }^{85}$. Ce métal s'applique à toutes les actions des hommes de la

79 W EST, op. cit., p. 177, «Hesiod presents the story not as an absolute truth». CARRIÈre, art. cit. 1986 et, 1991, insiste sur le fait qu'Hésiode ne croit pas à son histoire.

80 C. CALAME, «Mythe» et «rite» en Grèce : des catégories indigènes?, in Kernos, 4 (1991), p. 179-204. Curieusement, Calame n'envisage pas le cas du vers 106 des Travaux.

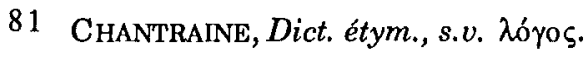

82 Voir en dernier J. RUDHARDT, Comprendre la religion grecque, in Kernos, 4 (1991), p. 47-59, notamment p. 56-58.

83 Platon, Cratyle, 398a.

$84 T h ., 764$.

$85 T h ., 726,733,750,811 ; 311$ (Cerbère). Autre emploi au sujet de Thanatos, Th., 764. Au sens propre, $O p ., 493$. 
troisième race, celles qui ont quelque rapport matériel avec lui, comme l'usage des armes, et celles qui n'en ont aucun. Dans ce cas il s'agit d'une qualification métaphorique destinée à marquer entièrement cette race du sceau des Enfers dont ils sont les premiers clients. Le bronze est le métal connoté le plus négativement chez Hésiode. Même le fer se trouve en quelques emplois neutres, de même que les hommes de la dernière race voient présentement quelques biens mêlés à leurs maux.

À l'inverse l'or est un métal totalement positif, associé à la beauté et à la valeur; épithète favorite d'Aphrodite ${ }^{86}$, il se trouve tout désigné pour caractériser l'époque où régnait l'harmonie entre les hommes et les dieux. La valeur de l'argent est moins aisée à saisir, parce qu'il n'y a, outre ceux qui figurent dans le mythe des races, que deux emplois de

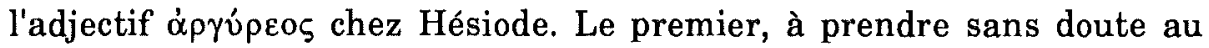
sens propre, concerne la demeure de Styx, "que de tous côtés des colonnes d'argent dressent vers le ciel» bien que la déesse «habite loin des dieux» ${ }^{87}$. Le second, métaphorique, s'applique aux tourbillons de son père, Océan, qui "s'enroule autour de la terre et... de la mer» ${ }^{88}$. Dans les deux cas, l'argent est associé au tracé des limites du monde : le cours d'Océan enserre les zones habitées par les hommes; les colonnes de la maison de Styx mesurent la distance qui la sépare du ciel. L'enclume de bronze, parcourant en un temps égal les distances du ciel à la terre et de la terre au Tartare ${ }^{89}$, suit la même trajectoire, mais dans un contexte où se marquent exclusions et mises à l'écart, puisqu'il s'agit de la précipitation au gouffre des Titans vaincus, alors que Styx au contraire, garant du serment des dieux, reçoit à ce titre la visite d'Iris, venant puiser son eau depuis l'Olympe ${ }^{90}$. Le bronze connote des changements d'état et des séparations irréversibles; l'argent est associé à la notion de limite et à celle de la distance en tant qu'elle n'est pas infranchissable. Il convient bien pour figurer l'état de la seconde race, à moitié séparée des dieux.

Le symbolisme des métaux figure donc le statut de l'humanité dans un monde en évolution. C'est tellement vrai que, lorsque le poète n'a pas

$86 T h ., 822,962,975,1005,1014$.

87 Th., 777-779.

$88 T h ., 791$.

89 Th., 722-725.

90 Th., 717-720 et 784-787. 
à sa disposition de métal susceptible de décrire le statut des héros, il s'en passe sans que la cohérence de l'ensemble soit anéantie.

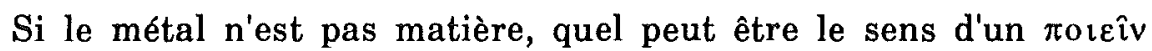
s'exerçant sur des objets, au sens concret et grammatical du terme, qui ne sont déterminés que métaphoriquement ? Ce "faire», inévitablement entraîné vers l'objet dans lequel il s'investit, ne concerne ainsi qu'une représentation, et non une présentation de la genèse des hommes. À l'appui de cette interprétation, on peut retenir l'absence de tout verbe «faire» en ce qui concerne la race de fer. De même que les héros se voient, sans grand dommage pour l'architecture de l'ensemble, dénués de toute référence métallique, de même les hommes de fer ne sont pas «fabriqués» par les dieux, et on s'en avise à peine ${ }^{91}$. Il est à noter que ces deux lacunes symétriques affectent, chacune pour leur part, les deux dernières races. Tout se passe comme si, les trois premières s'étant appuyées sur les deux motifs solidaires de la fabrication et du métal pour mettre en place un contenu de signification qui, lui, est essentiel, la quatrième et la cinquième races pouvaient se permettre de gommer ce qui n'est pas à retenir, c'est-à-dire la fiction à visée pédagogique.

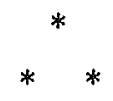

En somme, Hésiode utilise plusieurs matrices comme "moyens", selon les termes de J.-P. Vernant : le mythe, probablement emprunté, de la création successive de races métalliques, la structure trifonctionnelle, des schémas logiques et narratifs divergents 92 . Il en fait "savamment" un montage, dont il sait qu'il ne dit pas la vérité et qu'il

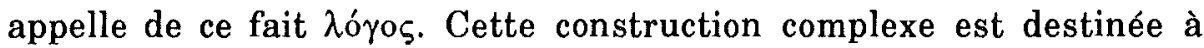
affermir la structure du mythe jusqu'à ce qu'elle tienne solidement et qu'on puisse, dans les deux dernières races, abandonner tour à tour les parties constitutives de ce coffrage pour ramener à l'essentiel : très

91 Seuls J. FONTENROSE, art. cit., et J. RUDHARDT, art. cit. (1981), accordent pleine valeur à cette lacune, moins remarquée que l'absence de métal pour caractériser les héros.

92 On pourrait évidemment intégrer, dans ces moyens littéraires destinés à structurer le mythe, la hiérarchie des puissances surnaturelles chère à Goldschmidt. Mais j'ai dit plus haut mon peu d'enthousiasme pour cette interprétation. 
proches à l'origine, dieux et hommes mortels sont désormais séparés sans qu'il soit pour autant fatal que cette séparation aille jusqu'à la rupture. Le vers 108 donne la clé de l'histoire. Il est le seul qui énonce la vérité à «se mettre en l'esprit». Tout le reste est littérature.

Rue des Hautes Bornes, 31,

Marie-Christine LECLERC

F - 94310 ORLY 